\title{
Assessing Clinical Outcomes Post Modified Laparoscopic Gastrostomy in Children: a Case Control Study
}

Hussein Naji ( $\nabla$ hussein.naji@mediclinic.ae)

Mediclinic Parkview Hospital

Aafia Mohammed Farooq Gheewale

Mohammed Bin Rashid University of Medicine and Health Sciences College of Medicine https://orcid.org/0000-00024056-0120

Ebtesam Safi

Mohammed Bin Rashid University of Medicine and Health Sciences College of Medicine

\section{Research article}

Keywords: Laparoscopic gastrostomy, Postoperative complications, U suture technique, Seldinger technique, Button, Children

Posted Date: July 19th, 2021

DOl: https://doi.org/10.21203/rs.3.rs-728208/v1

License: @ (i) This work is licensed under a Creative Commons Attribution 4.0 International License. Read Full License

Version of Record: A version of this preprint was published at BMC Surgery on February 21st, 2022. See the published version at https://doi.org/10.1186/s12893-022-01515-0. 


\section{Abstract}

\section{Background}

Gastrostomy has become a common surgical procedure within the pediatric population with feeding difficulties and nutritional issues. In the aims of improving clinical outcomes, this research targets to compare the rate of complications of two different laparoscopic techniques of a gastrostomy button placement in a pediatric population: A combination of modified U-stitches and seldinger technique laparoscopic gastrostomy (MLG) versus the standard laparoscopic gastrostomy (LG).

\section{Methods}

Eighty-nine children were recruited for this retrospective case control study that assesses the surgical outcomes of a novel MLG, being the cases to the standard LG in children which are the controls. The main outcome measure is the rate of postoperative complications encompassing dislodgement of gastrostomy button, leak around button, local infection, and development of granulation tissue post-surgery which is compared between the two population groups.

\section{Results}

The p-value of the study was shown to be 0.03 proving a statistical significance between the complication rates.

\section{Conclusion}

As a result, the modified U-stitches laparoscopic gastrostomy has a lower rate of complications in comparison to the standard laparoscopic gastrostomy making it a better technique for gastrostomy placement in children.

\section{Background}

Gastrostomy is a surgical procedure in which a feeding tube or a button is placed through the abdominal wall into the stomach in aims of long term nutritional support or administration of medications [1]. A gastrostomy is advised in patients that are unable to eat normally, for which the most common reasons are neurological causes, anatomical causes or obstruction of the esophagus. The determining factor of how long one is unable to eat poses different options for the patient; wherein general enteral feeding using a nasogastric tube is recommended when the patient is unable to eat normally for at least 7-14 days. Moreover, if the patient is unable to eat normally for more than 30 days, a gastrostomy as a recommendation goes higher up in the list [2].

With the advancement of minimal invasive surgeries, newer modifications and techniques have developed including percutaneous endoscopic gastrostomy, percutaneous radiologic gastrostomy and laparoscopic gastrostomies [3]. While all methods have varying complications, efforts in constantly improving patient outcome are being made.

Gastrostomy has become more common and widely accepted over the years in both infants and children. The surgery is done for children with feeding difficulties, malnourishment and other complex medical conditions in which the patient requires long-term enteral nutrition which has its obvious advantages over parenteral nutrition. Percutaneous endoscopic gastrostomy (PEG) was first introduced in the 1980s and quickly gained popularity [4]. According to the European Society for Clinical Nutrition and Metabolism (ESPEN), gastrostomy tube is indicated in patients who require enteral feeding for more than 2 weeks [5]. The goal of this is to prevent significant weight loss, nutritional deficiencies, assist with growth in children and improve their quality of life.

In addition to PEG and with ongoing advances, LG became more available. It is a minimally invasive procedure where the gastrostomy button can be placed directly (instead of a feeding tube), with shorter hospital stay, quicker recovery and fewer complications [3]. One meta-analysis study proved that the risk of major complications was higher in PEG than in LG with a 
95\% confidence interval of $1.90-7.81$ and a p value $<0.0002$, making it the preferred method for gastrostomy tube placement in children [6]. In addition, the risk of accidental gastro-enteric fistula development which occurs in $1.27 \%$ of PEG is much lower in LG [7].

The standard laparoscopy technique allows for easy placement of the gastrostomy with direct viewing and manipulation of the stomach yielding a minimized risk of unintentional visceral injury [8]. Before an LG procedure, the site of the gastrostomy is marked on the left upper quadrant below the costal margin to prevent pressure sores. The peritoneal cavity is opened through a sub-umbilical incision according to Hasson technique. Pneumoperitoneum is achieved by inflating carbon dioxide through the $5-\mathrm{mm}$ umbilical port at a rate of $1-3 \mathrm{~L} / \mathrm{min}$ until an intra-abdominal pressure of $8-10 \mathrm{mmHg}$ is obtained. Abdominal exploration is completed using a 5-mm (30-degree) telescope through the umbilical port. Another 5 -mm port is then introduced under direct vision over the designated site for the tube placement. The gastric wall is then grasped with a 5$\mathrm{mm}$ laparoscopic Babcock forceps and brought through the port site while simultaneously removing the trocar and decreasing the pneumoperitoneal pressure to $0 \mathrm{mmHg}$. Once exteriorized, the gastric wall is fixed to the anterior abdominal fascia with four sutures. The incision is sometimes enlarged up to an additional $1 \mathrm{~cm}$ for placement of the sutures. A gastrostomy is opened at the center of the sutures by diathermy. A balloon gastrostomy button is then inserted over a probe. Pneumoperitoneum $(10 \mathrm{mmHg})$ is recreated for control of the location of the button and the stomach.

While all methods have varying complications, efforts in constantly improving patient outcomes are being made. In line with the continued regard towards improvement, a novel technique was introduced in our pediatric surgery department with a modified U-stitches laparoscopic gastrostomy. This modification consists of a combination of hidden U-stitches placed under direct vision of laparoscopy to anchor the stomach to the abdominal wall and using Seldinger technique to insert the gastrostomy button.

The aim of this research is to compare the rate of complications of two different laparoscopic techniques of a gastrostomy button placement in a pediatric population: A combination of modified U-stitches and Seldinger technique laparoscopic gastrostomy versus the standard laparoscopic gastrostomy. The modified U-stitches technique applied to laparoscopic gastrostomy is expected to yield a decreased number of complications in comparison to the standard laparoscopic gastrostomy in children.

\section{Methods}

\section{Operative technique/Procedure MLG}

Patient is positioned supine on the surgical table under anesthesia. A $5 \mathrm{~mm}$ camera port is used to access the peritoneum according to Hasson technique through a subumbilical incision. A 3-mm laparoscopic grasper is introduced directly in the upper left quadrant (between the umbilicus and the costal margin). The anterior wall of the stomach is grasped near the greater curvature away from the pylorus to avoid any gastric outlet obstruction. A 2:0 Vicryl ( $1 / 2$ circle Taper Point Plus $44 \mathrm{~mm}$ Needle) is used to place an anchoring U-stitch through the abdominal wall and then through the full thickness of the gastric wall under direct vision with laparoscopic guidance and lastly going outside through the abdominal wall. This $U$ stitch is placed medial to the position of the grasper. Another $U$ stitch is going to be placed in the same way but lateral to the position of the grasper. The grasper is then removed while the stomach is anchored by the $2 \mathrm{U}$ stitches. A puncture needle (18-G) is inserted between the $U$ stitches through the same opening on the abdominal wall that was used for the grasper and then pushed under direct laparoscopic vision through the gastric wall to the stomach (Fig. 1a-1b). A guide-wire is then introduced through the puncture needle to the stomach. The needle is removed leaving only the guide-wire in place (Figs. 1c-1e). A telescoping dilator with a built-in peel-way external sheath is slowly introduced over the guide-wire (according to Seldinger technique) in a step-by-step telescoping manner (Figs. 1f-1h). After sufficient dilation, the dilator is removed and an over-thewire stoma measuring device was inserted to determine the appropriate length of the gastrostomy button to be placed. Next, the dilator is reinserted and the peel-away external sheath is introduced into the gastric lumen. The dilator is then removed, 
leaving only the external sheath with the guide-wire in place. A suitable size balloon gastrostomy button with an appropriate length shaft is inserted into the external sheath over the guide-wire and the external sheath is slowly peeled off. The guidewire is removed to leave only the gastrostomy button in place (Figs. 1i-1j). Each $U$ stitch is tied subcutaneously after pulling its 2 arms by a Mosquito artery forceps introduced in the subcutaneous space. The $2 \mathrm{U}$ stitches will ensure fixation of the stomach to the abdominal wall while the button's balloon will secure the button in its place and prevent its dislodgement.

\section{Data collection}

The study was approved by the Mediclinic Middle East Research and Ethics Committee (MCME.CR 161. MPAR. 2020) and Dubai Scientific Research Ethics Committee (DSREC), DHA (DSREC-01/2021_19). This is a retrospective analysis where data collected from the electronic medical records of all children who underwent laparoscopic gastrostomy button placement in a period of 8 years. The patients were divided into 2 groups depending on the type of surgical procedure.

1. The first group (cases) consisted of 48 patients who were operated with the newly introduced modified laparoscopic gastrostomy (MLG) by integrating the hidden $U$ stitches and Seldinger techniques for placement of the button.

2. The second group (control) consisted of 41 patients who were operated with the standard laparoscopic gastrostomy button placement $(\mathrm{LG})$.

The demographic data (age, sex and weight of patients were studied together with indications for gastrostomy, comorbidities, operative time and post-operative complications including leakage, infection, granuloma formation and dislodgment of the button. The two groups were followed up for a period of one year (at 1, 6 and 12 months).

Inclusion criteria:

All children who underwent a laparoscopic gastrostomy tube placement in our pediatric surgery department during the study period were included.

Exclusion criteria:

1. Children who underwent placement of gastrostomy button or tube via an open surgical method or PEG.

2. Children who needed a concomitant operation (like fundoplication) at the time of laparoscopic gastrostomy tube placement.

3. Two patients who underwent a LG but failed to attend during the follow up as they left the country for good during the follow up period (after 3 weeks and 6 weeks following the surgery).

\section{Statistical Analysis}

Statistical analysis was conducted using Microsoft Excel. Our null hypothesis of this study is that there is no difference in complications between the two surgical techniques. The two variables consisted of the two surgical techniques under which five complications were compared. The complications described were dislodgement of button, leak around button, redness and discharge around the button, local infection that required admission, and development of granulation tissue as is shown in Table 2. Percentages were yielded for each complication that reflects its rate for each surgical technique. With the application of data analysis on Microsoft Excel, we were able to find the p-value by using each variable on the $x$ and $y$-axis separately. The modified U-stitches $L G$ was inserted into the $Y$ input range while the $L G$ was inserted into the $X$ input range. By using the t-test and Mann-Whitney test, the p-value as a result, is 0.03 which rejects the null hypothesis proving a statistical significance to the study. Furthermore, the standard error is 0.07 which positively reflects on the sample population of this study, denoting a more accurate representation of the wider population.

\section{Results}


A total number of 89 children were enrolled in the study. The median age was 1.5 years (ranged between 1 month to 17 years), with no major intraoperative complications. There were no procedure related deaths nor was there any necessity to switch to open surgery. The median operating time was 55 minutes for the $L G$ and 56 minutes for the MLG as shown in Table 1. The postoperative complications are summarized in Table 2 with the most common complication for both procedures being formation of granulation tissue as seen in 15 patients (31\%) in group one versus 12 patients (29\%) in group two. Leakage around the button was markedly less in the second group (4\% versus $15 \%$ ).

The overall rate of complications however was still less with the MLG group. This is proven with the p-value $0.03(<0.5)$ resulting in a significant difference. While the study stands internally valid to its sample population, it also is externally valid with the evidence of a low standard error (0.07), denoting a close relationship between the sample and wider population.

Table 1

Age of patients and the median operating time.

\begin{tabular}{|lll|}
\hline & Laparoscopic Gastrostomy (LG) & Modified U-stitches LG \\
\hline No. of patients & 41 & 48 \\
\hline Median age & 1.5 years (range 1 month-17 years & 1.5 years (range 1 month-14 years) \\
\hline Median operating time & 55 minutes (range 46-94) & 56 minutes (range 3-112) \\
\hline
\end{tabular}

Controls: patients undergoing the standard laparoscopic gastrostomy

Cases: patients undergoing the modified U stitch $L G$.

Table 2

Summary of the postoperative complications of the two different procedures

\begin{tabular}{|lll|}
\hline Complications & Laparoscopic Gastrostomy (LG) & Modified U-stitches LG \\
\hline Number of patients & 41 & 48 \\
\hline Dislodgment of button & $2(5 \%)$ & $1(2 \%)$ \\
\hline Leak around button & $6(15 \%)$ & $2(4 \%)$ \\
\hline Redness and discharge & $8(20 \%)$ & $12(25 \%)$ \\
\hline Development of granulation tissue & $12(29 \%)$ & $15(31 \%)$ \\
\hline Local infection that required admission & $2(5 \%)$ & $2(4 \%)$ \\
\hline
\end{tabular}

\section{Discussion}

Laparoscopic gastrostomy has many overall advantages for children who require long-term enteral nutrition. The surgeon has a better visual field thereby lowering the risk of perforation of hollow viscous and vascular injury. Laparoscopic visualization avoids accidental gastro-enteric fistula formation which is encountered in $1.27 \%$ of PEG [7]. Placement of a button directly is very helpful to the patient and much easier to handle than tube gastrostomy.

The rate of postoperative complications were significantly less in the MLG group particularly leakage around the button and dislodgment of the button making this modified technique an enhanced surgical option for gastrostomy placement.

The $U$ sutures fixate the stomach to the abdominal wall in an efficient way with minimal handling of the gastric tissue as the stomach remained inside the abdomen all the time and never needed to be exteriorized. The same stitches will be buried under the subcutaneous tissues later on giving wonderful cosmetic results; particularly with the use of a $3 \mathrm{~mm}$ incision on 
the abdominal wall and then introducing the dilators according to Seldinger technique with minimal damage to the tissues. This soft handling of tissues and small incision is the reason for reduction of leakage around the button.

This modified technique of gastrostomy is simple, effective and takes approximately the same operative time as the standard surgery. Improving quality of life after surgeries is an ongoing goal for surgeons; hence placement of the button directly with minimal complication rate and providing a good cosmetic result is giving an advantage of MLG over the standard LG.

\section{Conclusions}

MLG is a less invasive surgical procedure compared to the standard LG with a significant decrease in post-operative complications in the studied sample. The modified laparoscopic gastrostomy technique (MLG); a combination of $U$ stitches and Seldinger technique, used in this study is safe and has proven to be advantageous in terms of excellent fixation of the stomach to the abdominal wall with minimal handling of stomach and favorable cosmetic results.

\section{Abbreviations}

MLG: Modified U-stitches and seldinger technique laparoscopic gastrostomy

LG: Iaparoscopic gastrostomy

PEG: Percutaneous endoscopic gastrostomy

ESPEN: European Society for Clinical Nutrition and Metabolism

\section{Declarations}

\section{Ethics approval and consent to participate}

Individual patient consent was waived due to the study being a retrospective study. The study was approved by the DHA (CP_7.1.02_F02) and MCME ethical committee (MCME. R 161. MPAR. 2020) Regional Ethics Review Board (reference No. 2011/1234-31/4). All patient information will remain anonymous hence this study doesn't pose any serious ethical considerations.

\section{Consent for publication}

Individual patient consent was waived due to the study being a retrospective study

\section{Availability of Data and Materials}

Due to patient confidentiality, data is not publicly available

\section{Competing Interests}

The authors declare no conflict of interest.

\section{Funding}

This research received no external funding.

\section{Author Contributions}


Conceptualization, H.N.; methodology, HN, A and E.; validation, H.N.; formal analysis, A and E. and H.N.; data curation A and E. and H.N.; writing-original draft preparation, $A$ and $E$ and H.N.; writing-review and editing, $A$ and E. and H.N.; supervision, H.N.; All authors have read and agreed to the published version of the manuscript."

\section{Acknowledgements}

Not applicable

\section{References}

1. Merli L, De Marco E, Fedele C, Mason E, Taddei A, Paradiso F et al. Gastrostomy Placement in Children: Percutaneous Endoscopic Gastrostomy or Laparoscopic Gastrostomy?. Surgical Laparoscopy, Endoscopy \& Percutaneous Techniques [Internet]. 2016 [cited 7 June 2021];26(5):381-384. Available from: https://journals.Iww.com/surgicallaparoscopy/Fulltext/2016/10000/Gastrostomy_Placement_in_Children_Percutaneous.8.aspx

2. Lo“ser C, Aschl G, He'buterne X, Mathus-Vliegen E, Muscaritoli M, Niv Y et al. Consensus Statement; ESPEN guidelines on Artificial enteral nutrition - percutaneous endoscopic gastrostomy (PEG). Clinical Nutrition [Internet]. 2005 [cited 7 June 2021];24(5):848-861. Available from: https://www.clinicalnutritionjournal.com/article/S0261-5614(05)00106$8 / p d f$

3. McSweeney M, Smithers C. Advances in Pediatric Gastrostomy Placement. Gastrointestinal Endoscopy Clinics of North America [Internet]. 2016 [cited 7 June 2021];26(1):169-185. Available

from: https://www.sciencedirect.com/science/article/abs/pii/S1052515715000902?via\%3Dihub

4. Glasson E, Wong K, Leonard H, Forbes D, Ravikumara M, Mews C et al. Evolving Trends of Gastrostomy Insertion Within a Pediatric Population. Journal of Pediatric Gastroenterology \& Nutrition [Internet]. 2018 [cited 7 June 2021];67(5):e89e94. Available from: https://journals.Iww.com/jpgn/Fulltext/2018/11000/Evolving_Trends_of_Gastrostomy_Insertion_Within_a.9.aspx

5. Rahnemai-Azar A, Rahnemaiazar A, Nagshizadian R, Kurtz A, Farkas D. Percutaneous endoscopic gastrostomy: Indications, technique, complications and management. World Journal of Gastroenterology [Internet]. 2014;20(24):7739. Available from: https://www.wjgnet.com/1007-9327/full/v20/i24/7739.htm

6. Sandberg F, Viktorsdóttir M, Salö M, Stenström P, Arnbjörnsson E. Comparison of major complications in children after laparoscopy-assisted gastrostomy and percutaneous endoscopic gastrostomy placement: a meta-analysis. Pediatric Surgery International [Internet]. 2018 [cited 7 June 2021];34(12):1321-1327. Available from: https://link.springer.com/article/10.1007/s00383-018-4358-6

7. Lantz M, Hultin Larsson H, Arnbjörnsson E. Literature Review Comparing Laparoscopic and Percutaneous Endoscopic Gastrostomies in a Pediatric Population. International Journal of Pediatrics [Internet]. 2010;2010:1-4. Available from: https://www.hindawi.com/journals/ijpedi/2010/507616/

8. Jones V, La Hei E, Shun A. Laparoscopic gastrostomy: the preferred method of gastrostomy in children. Pediatric Surgery International [Internet]. 2007;23(11):1085-1089. Available from: https://link.springer.com/article/10.1007\%2Fs00383-007-2015-6

\section{Figures}




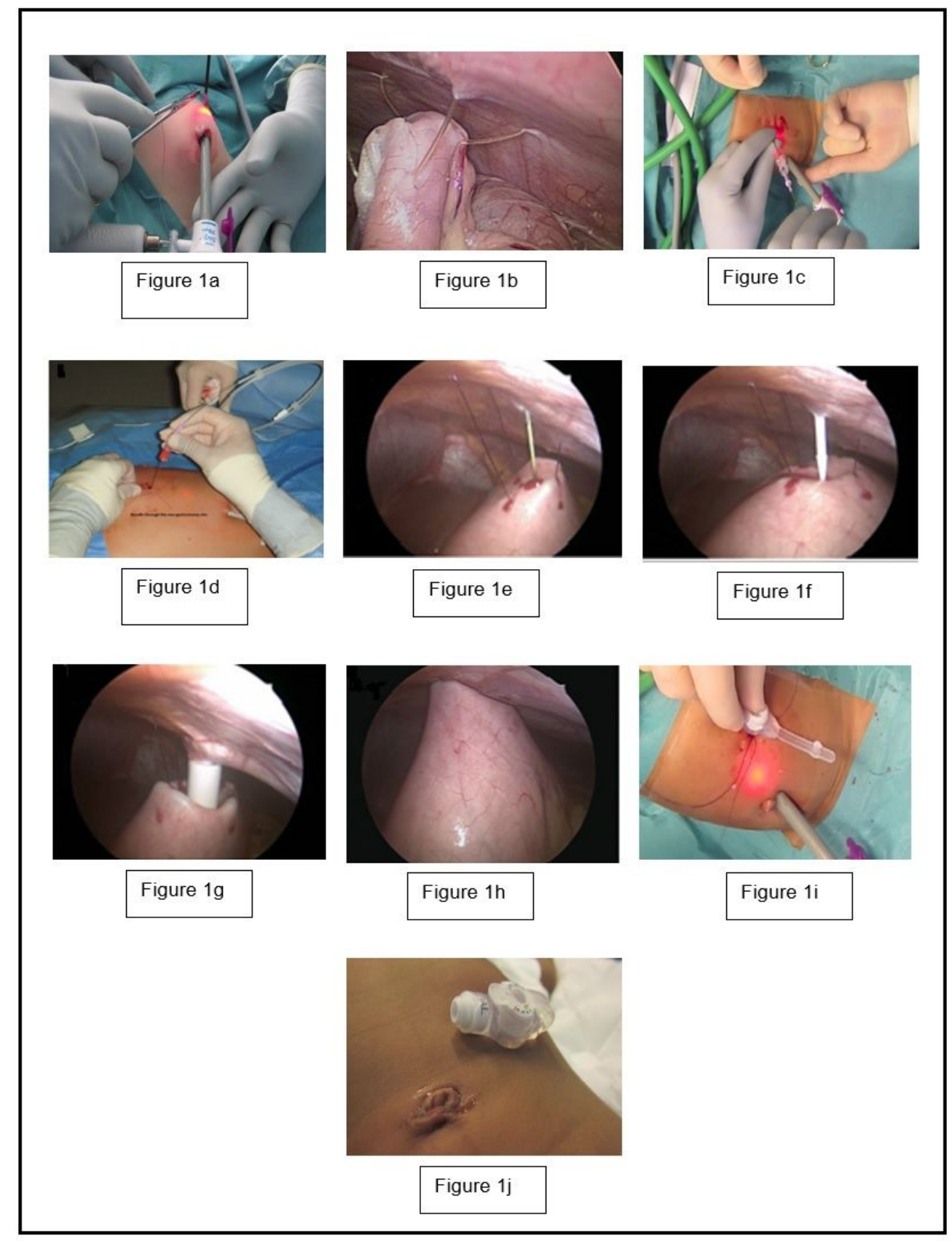

Figure 1

Technique of MLG 


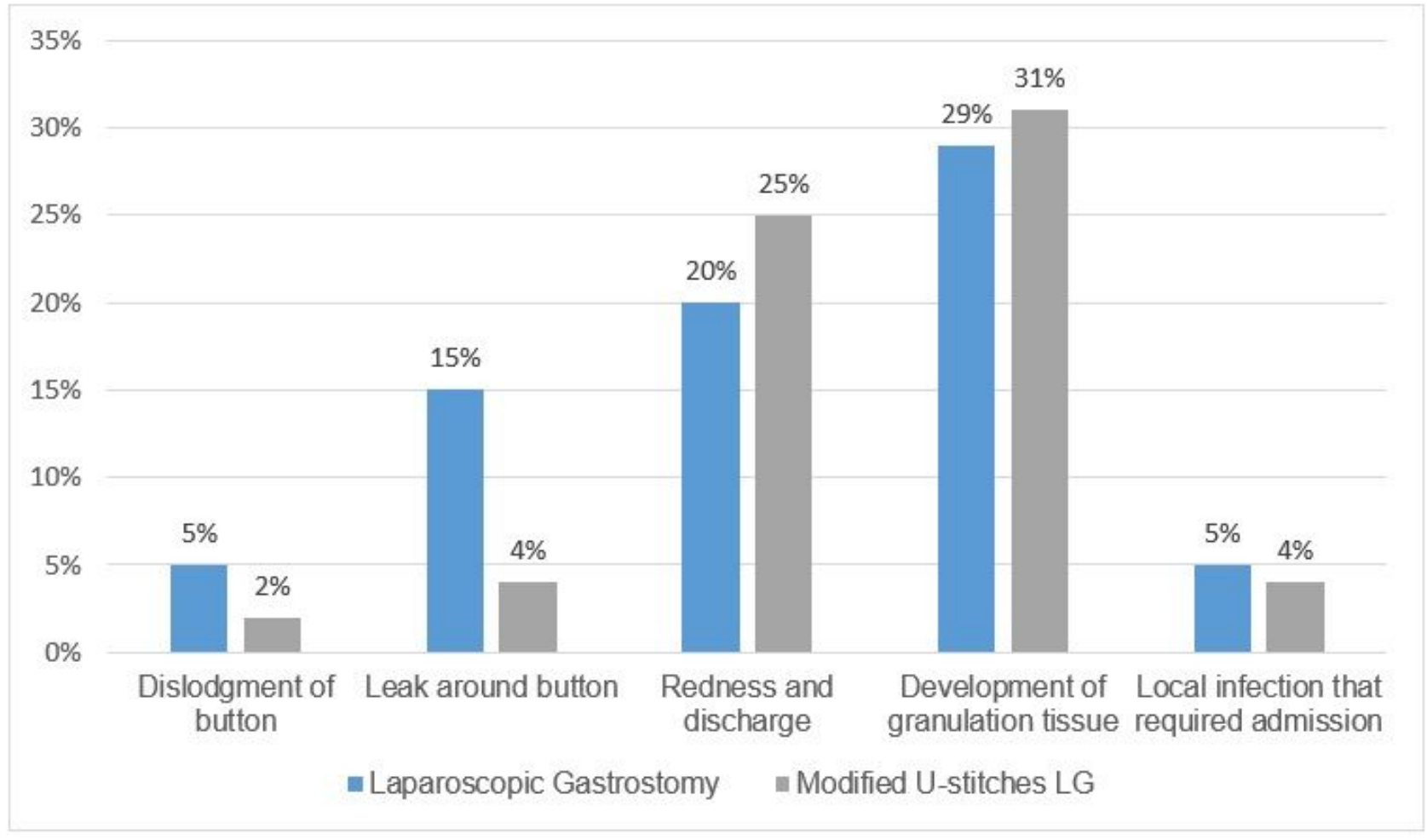

Figure 2

Bar graph representing the rate of complications 